\title{
Less invasive approach to degenerative lumbar deformity surgery
}

\author{
Max Aebi
}

(C) Springer-Verlag 2012

Keywords Adult scoliosis - Degenerative scoliosis · Degenerative disc disease - Discopathy - XLIF . Minimally invasive lumbar surgery

\section{Introduction}

The adult degenerative scoliosis has become a major concern in adult spine surgery. Since the disorder has many different expressions, the surgical treatment needs to be individualized to the patient's needs [1]. In an early stage of degenerative deformity limited surgery with a less invasive technique may be advantageous $[5,6]$. However, this technology needs specific training to avoid serious complications [4]. The surgeon who wants to perform the far lateral approach (XLIF) is well advised to study especially the anatomy of the psoas muscle and the relationship of the exiting nerve roots and the anatomy of the vessels in the lumbar spine [3].

\section{Case description}

The patient is a 58 -year-old woman who has been suffering from increasing low back pain for 2 years. The pain gets significantly worse while standing or lying in the same position. Walking diminishes her pain. The pain is frequently localised in the lower back and lumbosacral junction with irradiating

Electronic supplementary material The online version of this article (doi:10.1007/s00586-012-2206-2) contains supplementary material, which is available to authorized users.

M. Aebi $(\bowtie)$

Orthopaedic Clinic, Salem-Spital, Bern, Switzerland

e-mail: max.aebi@MEMcenter.unibe.ch along both iliac crests, extending to the buttocks and the thighs. It is primarily posterior and of unspecific character.

The patient has tried a number of conservative treatment modalities including pain medication, physiotherapy, chiropractic treatments and traditional Chinese Medicine. None have produced any long-term success. By the time the patient visited our clinic, she had already received regular doses of morphine medication, as well as pain pads. The patient was demoralised by the pain, unable to work and socially disintegrated.

The clinical examination was uneventful, with normal neurological findings in both legs, a tendency to bend forward in the sagittal balance, with a slight rotation to the right side. She could bend forward to a fingertip-floordistance of $10 \mathrm{~cm}$. Standing and sitting in the same position was painful.

The patient's native lumbar X-rays show a significant narrowing of the disc space L2-L3 and, to a lesser extent L3L4, with beginning of local kyphosis and right convex scoliosis, subchondral sclerosis L2-L3 as well as retrolisthesis of L2 towards L3. Under maximal flexion there is a certain reduction of the retrolisthesis and under maximal extension air inclusion in the disc L2-L3 is noted as an expression of a pathological motion. The MRI confirms a severe multilevel disc degeneration, with the segment L2-L3 most severely affected, as well as subchondral edema. Discography at the level of L3-L4 and L2-L3 initiated a concordant back pain.

The patient's history, the clinical findings as well as her moderate responsiveness to non-surgical treatment led to the decision for surgery.

\section{Surgical strategy}

In order to avoid damaging the paravertebral muscles, and to excise the pathological discs L2-L3 and L3-L4, a 
combined procedure was chosen: XLIF through a right far lateral access L2-L3, L3-L4 in left lateral position as well as a posterior transmuscular stabilisation L2-L4 with pedicle screws. The XLIF procedure was done with the Oracle cage system from Synthes, filled with demineralised bone matrix, and the pedicle fixation was performed with the Matrix MIS System.

\section{Postoperative information}

The patient was mobilised with a lumbar belt on the first day after surgery. Isometric exercises for the abdominal and paravertebral muscles were started immediately.

The postoperative examination showed a neurologically intact patient. The postoperative course was characterised by a difficult weaning off the patient's preoperative morphine medication.

3 months after surgery the patient's ability to perform daily routines were dramatically improved, and she experienced less pain than before surgery. The patient hopes to return to her job as a nurse.

\section{Discussion and conclusion}

The combination of a far lateral approach with lumbar disc excision and cage implantation with a secondary stabilisation by a posterior approach and pedicle fixation is a liable procedure in lumbar deformity surgery, as long as the spinal canal does not need to be explored. The posterior approach and the pedicle screw placement can either be done by a percutaneous procedure or with a skin incision and the subcutaneous transfascial screw placement, as demonstrated in this case, or a regular posterior median approach and exposure of the lumbar spine can even be considered. The latter procedure would also allow a formal spinal canal decompression. The percutaneous technique has a certain fiddling factor, specifically in a more than unisegmental fixation. In such cases, a formal skin incision may be helpful to expose the dorsal lumbar fascia and then have the pedicle screws as well as the rod positioned through a muscle splitting, as exemplified in this case.

The far lateral approach through a small incision can be made safer by using the neuromonitoring, when splitting the psoas muscle and preparing the targeted discs [2, 3, 5]. The overall blood loss and the surgical trauma appear to be less than in a conventional posterior surgery in combination with pedicle screws and TLIF or PLIF. Today several studies are under way to demonstrate that the advantages are also resulting in more favourable treatment costs and earlier return to the daily routine and work for the patient.

\section{References}

1. Aebi M (2005) The adult scoliosis. Eur Spine J 14(10):925-948

2. Dakwar E, Cardona RF, Smith DA, Uribe JS (2010) Early outcomes and safety of the minimally invasive, lateral retroperitoneal transpsoas approach for adult degenerative scoliosis. Neurosurg Focus 28(3):E8

3. Hu WK, He SS, Zhang SC, Liu YB, Li M, Hou TS, Ma XL, Wang J (2011) An MRI study of psoas major and abdominal large vessels with respect to the X/DLIF approach. Eur Spine J 20(4):557-562

4. Isaacs RE, Hyde J, Goodrich JA, Rodgers WB, Phillips FM (2010) A prospective, nonrandomized, multicenter evaluation of extreme lateral interbody fusion for the treatment of adult degenerative scoliosis: perioperative outcomes and complications. Spine 35(26 Suppl):S322-S330

5. Mundis GM, Akbarnia BA, Phillips FM (2010) Adult deformity correction through minimally invasive lateral approach techniques. Spine 35(26 Suppl):S312-S321

6. Ozgur BM, Aryan HE, Pimenta L, Taylor WR (2006) Extreme lateral interbody fusion (XLIF): a novel surgical technique for anterior lumbar interbody fusion. Spine J 6(4):435-443 